\title{
Voice Search Technology: An Overview
}

\section{Praveen Kumar Donepudi}

Senior Architect, IPM Practice Division, Cognizant Technology Solutions, Pittsburgh, Pennsylvania, USA

\begin{abstract}
Voice search technology is not new. It's been there since Audrey came out. But now it is increasing. People are using voice assistants, and so far 420 million voice assistants have been sold. This is growing rapidly because artificial intelligence integrated with voice search technology is more accurate and faster than text search. According to Google, its accuracy has improved to $95 \%$. In short, it's an old technology that's gaining popularity with the rise of mobile phone voice assistants. In this study, the researcher has introduced different aspects and practical implications of voice search technology.
\end{abstract}

Keywords: Voice Search, Search Technology, Artificial Intelligence, Business Learning

\section{INTRODUCTION}

People these days are always looking for quick and easy solutions that can make multitasking more effective. This makes speech-to-text conversion an ideal candidate. Also, those who are not good at typing or who find typing inconvenient find this option very attractive. Advances in technology and low error rates in voice-to-text conversions are changing the way people search online. As more and more people search using voice commands, it makes sense for digital marketers to be able to search content from all types of requests, including voice search, questions, and queries. Websites that were previously optimized for text-based searches and search criteria should now be optimized for voicebased queries.

\section{What Is Voice Search?}

Voice search is basically an advanced technology approach that uses voice-enabled commands to perform a search in an internet browser, phone, or any application. It basically contains a short keyword query for open domains to get information on the internet. Google Voice Search, Siri, Alexa are voice assistants that perform voice search. Meredith Broussard, Data Journalist, New York University Professor

This is basically a kind of dialog system with rounds of dialogue. You can also clarify the system for searching. This will give you more accurate results.

In other words, voice search is an approach in which the technology uses a voice recognition system to understand what the speaker is saying with reasonable accuracy.

It may sound like a brand new technology, but originally it was here for a while. A program with a text-to-speech option is a perfect example of voice search. 


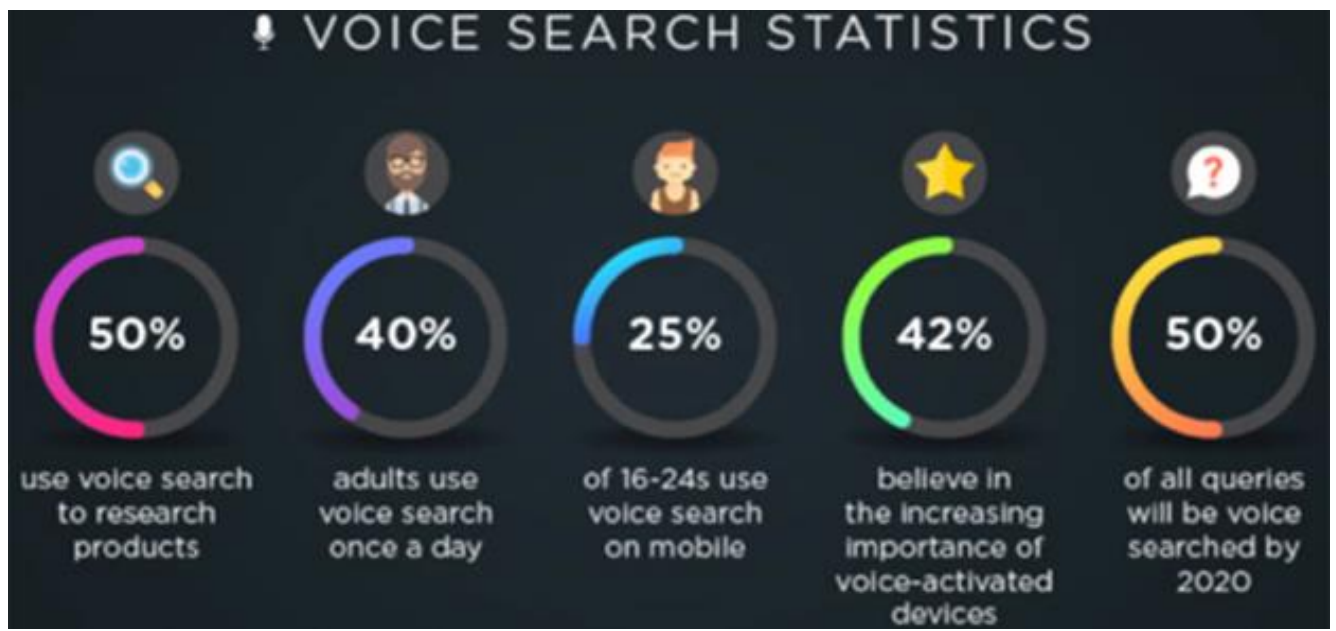

Why Is Voice Search Growing So Quickly?

Voice search is growing rapidly, and you're not the only one aware of it. We all embrace this trend and for good reason. There are three drivers who are responsible for their rapid success now and in the future. Alexander Radnicky, Research Professor, Carnegie Mellon Speech Group

The first reason is that it is 3.7 times faster than input. The faster the search, the faster the answer.

Second, it is ideal for portable electronic devices such as mobile phones and tablets. Total of the $60 \%$ mobile phone user search with voice.

Third, it's more convenient. Better than typing on the keyboard. In short, the use of voice search is no longer hyped. It's a trend and stays here. In fact, it's also starting to affect search engine SEO rankings.

\section{Voice Search and Other Spoken Dialog Technologies}

The table below compares the three types of technologies.

\begin{tabular}{|l|c|c|c|c|}
\hline & \multicolumn{2}{|c|}{ User input utterances } & \multicolumn{2}{c|}{ Target semantic representation } \\
\hline & Naturalness & Input space & Resolution & Semantic space \\
\hline $\begin{array}{l}\text { Form filling/directed } \\
\text { dialog }\end{array}$ & low & small & low & small \\
\hline $\begin{array}{l}\text { Form filling/mixed- } \\
\text { initiative }\end{array}$ & Low-medium & small & high & small \\
\hline Call routing & high & large & low & small \\
\hline Voice search & Medium-high & large & low & medium-large \\
\hline
\end{tabular}

\section{Why Is Voice Search Important?}

You may be looking for a reason to know how important this trend is to most people. You may not use it now, but see the stats below the market. These statistics tell you how everyone chooses it and when you should start using it.

- $\quad$ By 2020,50\% of all web queries will be voice search. 
- $50 \%$ of people use voice search when searching for products in their browsers and social media platforms.

- $40 \%$ of adults in the global community use voice search for regular Internet queries.

\section{What Does Voice Search Look Like?}

When you type in the Google search bar, you'll see a microphone symbol just inside the bar. You can click this symbol to actually talk about your query using keywords. But let's explain further through one clear example.

A search on the Google bar will bring up a box of interest. Google basically calls them feature snippets.

Each time you enter a question, Google derives the appropriate answer from the article via keywords and places it in the snippet. Mike Cohen, Google's Voice Technology Manager

Featured snippets come in three forms. One is a one-word answer, the second is a dataset or list, and the third is a paragraph. Google usually displays paragraphs and lists simply because it's very difficult to pull specific data from a dataset. This limits the user's search and makes them unaware of the current data on the Internet.

The biggest advantage of featured snippets is that you don't have to rank them first to include website paragraphs. If you are in the top 10 positions, you can jump over other sites and be on top of them.

Many web professionals call this area position zero.

\section{How Does Voice Search Work?}

Dr. Frank Ludzic, Toronto Rehabilitation Institute-UHN

Voice search is very easy to work with. Just read the results from the featured snippet data extracted from the article. So every time you ask a voice assistant, you'll get an answer from the information in the snippet of interest.

This is very beneficial for business-to-business transactions. When a website reaches a featured snippet, mobile and desktop users will primarily use voice search, improving their SEO rankings.

Voice search has many advantages if you have a website that looks like this:

- Brand awareness / awareness.

- Increased traffic to your website.

- Excellent click rate.

\section{How to Turn on Voice Search?}

- It's very easy to turn on and use voice search. If you have an Android tablet or smartphone, open the Google app.

- $\quad$ "Details" is displayed at the bottom right. Click on it and select Settings $>$ Audio.

- Turn on voice matching so that Assistance can access the microphone.

How to Turn Off Voice Search?

When you have to turn off the voice search you have to follow these:

- Go to "Settings" in the Google app. 
- Click the General tab.

- Under the Personal option, there is an Input and Language option. Click.

- Then tap GOOGLE OK detection.

- To turn it off move to the slider to the left.

- You have successfully turned off your Android mobile or tablet.

\section{Voice Search Optimization?}

Voice search optimization is basically a voice-based search engine optimization. It currently plays a major role in the SEO industry and influences the SEO rankings of each website. Spitch CEO Alexey Popov

Voice engine optimization is an approach that involves optimizing services, content, locations, websites, and company information, and can boost your SEO ranking through voice-results searches.

Search by voice optimization started in 2017. Currently used for quick answers. It's similar to SEO, but it's more competitive.

Unlike traditional searches, there are only three results. So if you're not in it, you're the last.

\section{How to Optimize for Voice Search?}

Search marketing is broad and it is very important to optimize voice search to get the most out of it. People want to know quickly and fortunately about special ongoing deals, packages, products, news, or any kind of information. They found a way to find it. Of course, by voice search. Bengomes, Google Search Director

Here are five major changes that need to be considered to optimize voice search:

\section{Keyword Research for Voice Search}

The first thing you need to know is that people speak differently. One may use words that the other does not. People ask questions in different ways, especially verbally. Therefore, you need to brainstorm all relevant questions about your business or the product that people may be looking at the answer.

There are many tools to help you get the best questions related to your brand. Answer the Public is a tool that helps you create as many questions as you can using specific keywords.

People Also Ask is a great Google option to help you get ideas for more questions.

\section{Site Structure and Content Development}

It should be available at all stages of customer support through voice search. People can ask anything about your company, and you should be able to answer them. Here are some points you can focus on when developing and curating better content for voice search:

- Consciousness.

- Rating.

- Interest.

- Customer support.

- Buy

- Loyalty. 
Try organizing your brainstormed queries and questions into different categories. This will help you map your customer journey properly. Kelly Davis, Mozilla Machine Learning Researcher

The most effective solution to this problem is to add the FAQ to every page of your website.

\section{- $\quad$ Structured Data Optimization}

The key to optimization is to make the content as relevant as possible to the query. Make sure Google can easily crawl the pages of your site. To do this, create a website map as soon as possible and submit it to the Google Console.

Another thing you can focus on is bots. Use a schema markup approach to help Googlebot understand your content in the best possible way.

Provides Googlebot with relevant information about products, brands, locations, contact information, and more.

You can also provide information about a variety of business-related content, articles, organizations, event seminars, recipes, and reviews.

\section{- Consider Creating Alexa Skills}

You can design your custom Alexa or Google Assistant skills to make your content more accessible to your Personal Voice Assistant. To make Google's actions even smoother, you'll need to customize your software for your voice assistant. Improve Google's actions to improve the experience for voice command users and enable them to interact with content and applications.

- Mobile-Friendly

Search engine marketing focuses on mobile-friendly content and search, not desktopfriendly. It is true that most voice searches are done from mobile phones. Therefore, when optimized for mobile-friendly search, it is actually optimized for voice search.

\section{VoICE SEARCH FOR E-COMMERCE}

Buying things by voice alone is a whole new way of shopping, and its popularity is skyrocketing. Shopping is the third most popular use of smart speakers (after playing music and gathering information). Almost a quarter of voice assistant users say they purchased using gadgets.

\section{There are two important factors that contribute to this surge in usage.}

1. Voice shopping is easier than sitting and searching on a screen-based device. For example, some large supermarkets have Alexa skills that allow you to add things to your shopping cart just by asking, significantly accelerating the normally cumbersome weekly grocery store.

2. Voice shopping is barrier-free. Instead of taking your phone out of your pocket, unlocking it, finding the app, and waiting for it to load, you can use voice technology to make a quick purchase. Limiting the amount of friction between the customer and the product is a great advantage of voice shopping. 


\section{Benefit your business}

Anh Ta is a PhD candidate in Business Sciences from the University of North Texas School of Information Technology and Decision Sciences. His current research interests include healthcare management, consumer environmentally friendly purchasing behavior, supply chain management, structural equation modeling and optimization.

1. Voice shopping is perfect for cross-selling. Voice is a very natural and relaxed way of interacting, so customers are much more likely to take you to special offers and product recommendations. Imagine a scene. If Alexa says, "Why don't you try a new chocolate cheesecake? It's only \$ 1 for this purchase," I'm trying to confirm my weekly shop order. Do you think no? Of course you don't!

2. Voice shopping helps you personalize your customer journey. You can gather a lot of information from how your customers sound. Are they men or women? Old or young? All this data helps to provide a more personalized, customized shopping experience and ensures that more people are back.

\section{Virtual Assistant Technology Drives Voice Search}

The adoption of voice search on mobile phones using voice assistants such as Siri and Google Assitant and the rise of the Internet of Things, the Internet of Things, are important drivers of the rise from voice to text and voice-based search styles. Currently, the most used personal voice assistance services are Google Voice Assistant, Siri from Apple Inc., Cortana from Microsoft, and Alexa from Amazon. Top 4 virtual assistants

1. Siri (Apple)

2. Google Now (Google)

3. Alexa (Amazon)

4. Cortana (Microsoft)

Apple was the first large company to equip mobile phones with personal voice assistants. Apple has added personal assistant and voice search technology to the iPhone and named it Siri. Google also came up with its personal voice assistant and introduced Google Now. Google Now is an app that aims to give you the information you need before asking a question, taking inspiration from your daily browsing history, phone usage, and even your location and geographic data. Microsoft recently debuted a new personal assistant, Cortana, with the Microsoft Windows 10 operating system. Taking it one step further, Amazon has developed an Amazon Echo device and a virtual assistant called Alexa. Alexa works with your smartphone to answer questions such as: Handle requests such as "Alexa, how's the weather this week?" Alexa, reschedule the meeting at 10am. "Search trends clearly have a big impact as the adoption of these items increases. Only a few years ago, voice-based searches were searched around the world. Leading sources have marked this as about $20 \%$ of all mobile searches currently used as voice search, which shows no signs of slowdown. Marketers have spent years looking for the perfect solution to improve their site's ranking on search engine results pages (SERPs).

In most cases, if your ad or site details appear on the first page of SERP, it's considered a win. Optimization is primarily focused on creating compelling copies to win clicks on target users while leveraging the right keywords. It doesn't have to be the first option on the results page, as users are accustomed to at least scrolling through all the results on the first search page. 


\section{Comparison of Data Collection Methods}

\begin{tabular}{|c|c|}
\hline Variables & $\begin{array}{ll}\text { - } & \text { Setup time } \\
\text { - } & \text { Training } \\
\text { - } & \text { Number of subjects } \\
\text { - } & \text { Observer's focusd } \\
\text { - } & \text { Encumbrance } \\
\text { - } & \text { Resulting data computer ready } \\
\text { - } & \text { Automatic error checking } \\
\text { - } & \text { Cost of system } \\
\end{array}$ \\
\hline $\begin{array}{l}\text { Penci } \\
\text { 1/Paper }\end{array}$ & $\begin{array}{ll}\text { - } & \text { Low } \\
\text { - } & \text { Low to medium } \\
\text { - } & \text { Limited to form/paper size } \\
\text { - } & \text { Split -subjects and data entry } \\
\text { - } & \text { Hands required } \\
\text { - } & \text { No } \\
\text { - } & \text { No } \\
\text { - } & \text { Low }\end{array}$ \\
\hline $\begin{array}{l}\text { Audio } \\
\text { Recordings }\end{array}$ & $\begin{array}{ll}\text { - } & \text { Low } \\
\text { - } & \text { Low to medium } \\
\text { - } & \text { Unlimited } \\
\text { - } & \text { Solely on subjects } \\
\text { - } & \text { Hands free } \\
\text { - } & \text { No } \\
\text { - } & \text { No } \\
\text { - } & \text { Low } \\
\end{array}$ \\
\hline $\begin{array}{l}\text { Keyboard } \\
\text { Assisted }\end{array}$ & $\begin{array}{ll}\text { - } & \text { Medium to high } \\
\text { - } & \text { Medium to high } \\
\text { - } & \text { Limited to keyboard mappings } \\
\text { - } & \text { Split -subjects and data entry } \\
\text { - } & \text { Yes } \\
\text { - } & \text { Yes } \\
\text { - } & \text { Medium to high } \\
\end{array}$ \\
\hline $\begin{array}{l}\text { Voice } \\
\text { Recognition }\end{array}$ & $\begin{array}{ll}\text { - } & \text { Low } \\
\text { - } & \text { Low to medium } \\
\text { - } & \text { Unlimited } \\
\text { - } & \text { Solely on subjects } \\
\text { - } & \text { Hands free } \\
\text { - } & \text { No } \\
\text { - } & \text { No } \\
\text { - } & \text { Low } \\
\end{array}$ \\
\hline PDA Based & $\begin{array}{ll}\text { - } & \text { Low to medium } \\
\text { - } & \text { Low } \\
\text { - } & \text { Limited to screen size } \\
\text { - } & \text { Split -subjects and data entry } \\
\text { - } & \text { Hands required } \\
\text { - } & \text { Yes } \\
\text { - } & \text { Yes } \\
\text { - } & \text { Low to medium }\end{array}$ \\
\hline
\end{tabular}


With voice-based search, all of this is changing. There are no longer multiple options offered by voice-based search that users consider before choosing the best one that seems to suit their needs, like traditional text search. In most cases, the first result you hear will satisfy the query, or you will try another query to change the first search result. What this means now is that even the second search result isn't enough. Much of this is related to the particular nature of voice search and the challenges presented to sites optimized for typed search.

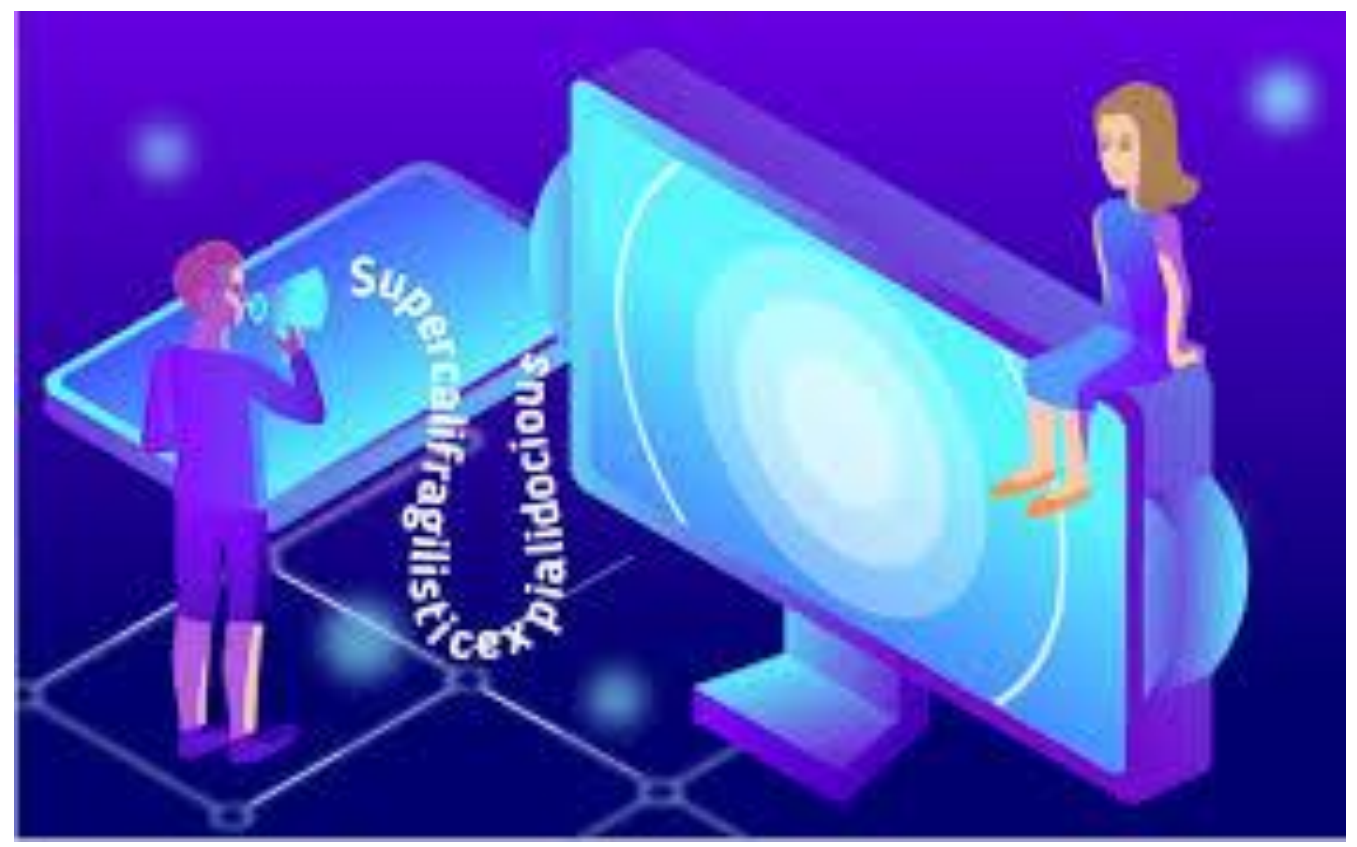

\section{Most Impressive Statistics on Voice-Based Search}

- According to GeoMarketing, 65\% of users of Google Home or Amazon Alexa can't even imagine going back to the days before using smart speakers.

- According to Google, having a voice-activated speaker and talking to it likes to talk to friends, $41 \%$ of users who own a voice-activated speaker say.

- According to GlobalWebIndex, 1 in 4 people aged 16-24 use voice-based search on mobile

- According to eMarketer, $70.6 \%$ of Americans who use voice-enabled speakers at least once a month in 2017 used Amazon Echo, and 23.8\% used Google Home.

- Of all voice-activated speaker owners, 39\% want to receive options that help them find business information, and $52 \%$ receive information about deals, sales and promotions from the brand. I would like to. Every Google.

- $\quad$ By 2020, half of all searches will be voice-based, according to comScore.

\section{Voice Search for Marketing}

Although the rules of the game are very different from traditional online campaigns, voice search has great potential as a marketing tool.

First, neither major brand voice assistant (Google Home / Amazon Alexa) allows paid advertising on the platform. There is a good reason for this. Advertising will discourage people from adopting technology and undermine the fragile trust that these companies have been working hard to build. 
One way to avoid this is to focus voice-driven marketing activities on brand building instead. We can easily create Alexa skills and Google "actions" to interact with your viewers in new and interesting ways.

There are many examples where this worked. For example, consider the fabric care brand Tide. Alexa's "Stain Remover" skill allows users to seek specific stain removal advice and receive step-by-step instructions (including tide products, of course).

One drawback of taking this route is that it requires a lot of hard work. Creating an Alexa skill or Google action is fairly easy, but the challenge is to make it reliable and functional enough that people want to use it over and over again.

Also, because the technology is still in its infancy, there is no guide to best practices for voice apps. So designing a voice app and measuring its success is a good guess.

Skills and actions may not have the same sales impact as direct online advertising, but like social media channels, they are a great way to build brand personality, awareness, and credibility.

Another marketing angle adopted by voice search technology is to play old SEO games. Unlike searching for answers on a device with a screen, a voice search device provides users with only the top search results, not just a few lists. This means that it's more important than ever to SEO-optimize your content and push it to the top of SERPs.

Voice search for marketing is clearly still in the works, but like all new attempts, there are plenty of rewards for companies that are willing to soak their feet in the water. Follow these tips to maximize your chances of success in your voice marketing efforts.

From an ease of use and SEO perspective, it's important to keep voice search results and applications conversational, understandable, and generally useful.

It is also important to answer the direct questions in the content concisely, and most voice search queries are explicit questions.

As more and more e-commerce and content websites offer voice search, users will expect this feature as standard.

Don't allow your website to be left behind. By investing in a site search solution that can provide voice search, your website will be available in the future for the voice-first revolution.

Challenges of Voice Search. Like other new technologies on the block, there are some toothy problems that companies must overcome if they want to penetrate the mass market with voice search.

Privacy concerns. Have you ever told a friend directly that you're thinking of booking a vacation just to find your Facebook feed full of travel ads?

It's creepy.

Facebook has repeatedly stated that it is "100\% not listening to our conversations", but is it really reliable when involved in many privacy scandals?

In a world where Google, Facebook and Apple already know us better than us, no one wants to be spy. Allowing tech companies to listen to our conversations is too far a bridge for many consumers. $79 \%$ of people are concerned about the privacy implications of using 
a voice search device. And the new generation is becoming more and more aware of these issues, with the youngest voice searchers (13-21) being very worried about voice search and privacy $515 \%$ more than the oldest $(65+)$. I feel that

As voice search becomes more prevalent, tech companies need to do more to calm consumer concerns and become more transparent about how voice data is used, processed, and stored. Google is already off to a good start and users can delete captured voice clips, but more needs to be done to build trust with consumers.

\section{Voice is still finding its feet on desktop}

Both Windows PCs and Apple Macs have Cortana and Siri, respectively, but the web browser itself still has very little voice search capability. Google Chrome is so far the only web browser with built-in voice search.

Until voice search is available on all devices and all browsers, tools will struggle to become a ubiquitous way to search the Web.

\section{Language support and regional accents}

Speech recognition has become fairly good at understanding American and British English accents, but most solutions are still inadequate when it comes to recognizing accents in other languages and regions.

This is not only annoying. Certain languages, such as Icelandic, are at risk of digital extinction because English dominates the Internet.

There is no doubt that this will eventually improve as the speech recognition database continues to collect more regional data samples. Still, it's a real problem that is currently blocking the investment of people with stronger accents on voice search devices.

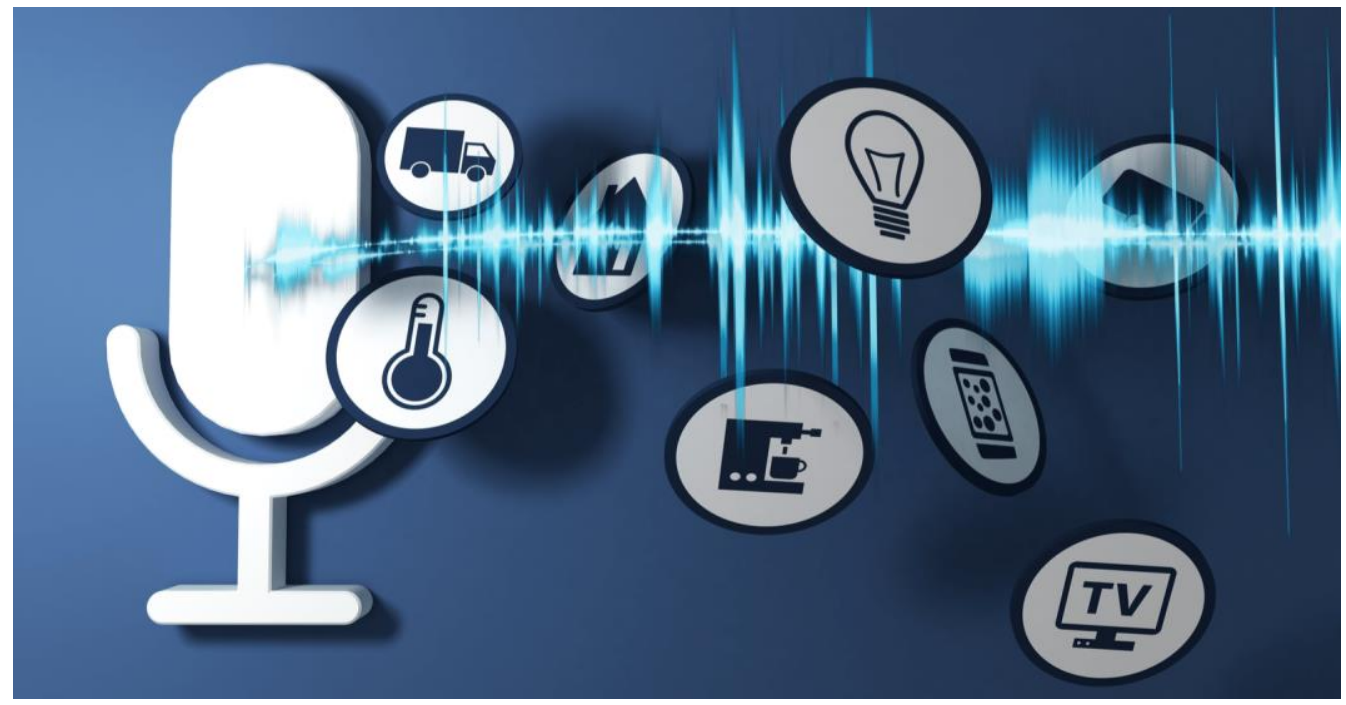

\section{VOICE SEARCH IMPACTS}

Half of the searches in 2020 will be voice-based due to the dramatic improvement in user experience and convenience. Victor Prybutok Ph.D., CQE, CQA, CMQ / OE, PSTAT® is a professor of decision science in the Department of Information Technology and Decision 
Science, and Dean and Vice President of the Toulouse Graduate School of the University of North Texas. .. He has written over 180 journal articles and over 250 conference presentations. Due to its high utilization, Google and other search engines are now increasingly focused on optimizing voice.

Search engine optimization or SEO is very important to users who are searching because they rank users according to the best information available to them.

What we're trying to say is that SEO has a huge impact on voice search. Voice-enabled searches have dramatically improved your website's SEO rankings.

This is how Search by voice impacts you:

- More natural language queries have been added.

- Instead of a text search, more questions will be asked.

- More local searches are being done.

- People are doing more action searches.

- Here are five steps to follow:

- Make your content easier to search by voice.

- Target long tail keywords due to the long voice search.

- Target Google's featured snippets.

- Optimize for natural language.

- Use metadata and structured data to help search engines understand you better.

\section{REFERENCES}

Digital Marketer Sound Off: Tips, Tactics, Tools, Predictions from 101 Digital Marketing SpecialistsMatt Tierra, Matt Tierra, May 31, 2018

Digital Marketing Analysis: Understanding Consumer Data in the Digital World --Chuck Hemann, Ken Burbary --Que Publishing, April 10, 2013

Digital Marketing Excellence: Online Marketing Planning, Optimization, Integration-Dave Chafei, PR Smith-Taylor \& Francis, March 31, 2017

Digital Marketing for Dummy-Ryan Dice, Las Henneberry-John Wiley \& Sons, January 17, 201

Digital Marketing Handbook: Step-by-Step Guide to Creating Websites for Sale --Robert W. Bly -Entrepreneur Press, April 17, 2018 B2B

Digital Marketing in the Zone: The Ultimate System for Digital Marketing Success --David Reske -Morgan James Publishing, June 9, 2017

Digital Marketing Strategy: An Integrated Approach to Online Marketing-Simon Kingsnorth, Kogan Page Publishers, May 3, 2016

Digital Marketing: A Practical Approach-Aran Charlesworth-Routledge, July 25, 2014

Digital Marketing: Marketing Directly to Businesses Using the Web--Michael Miller, Que Publishing, March 7, 2012

Experience: 7th Era of Marketing-Robert Rose, Carla Johnson-Content Marketing Institute

Fashion and Luxury Brand Digital Marketing Strategy-Ozuem, Wilson, Azemi, Yllka, IGI Global, October 31, 2017-

The Art of Digital Marketing: The Definitive Guide to Creating Strategic, Targeted, Measurable Online Campaigns-Ian Dodson, John Wiley \& Sons, April 4, 2016

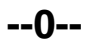


